\title{
Importancia del anestesiólogo en la prevención del dolor crónico
}

\section{Importance of the anesthesiologist in the prevention of chronic pain}

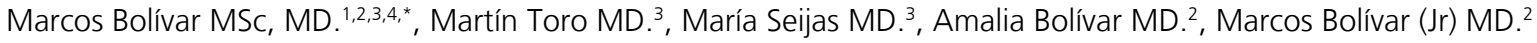 \\ Universidad Central de Venezuela (UCV). Caracas, Venezuela. \\ Hospital de Clínicas Caracas (HCC). Caracas, Venezuela. \\ Instituto Médico La Floresta (IMLF). Caracas, Venezuela. \\ 4 Grupo Médico Santa Paula. Caracas, Venezuela.
}

Fecha de recepción: 22 de febrero de 2021 / Fecha de aceptación: 20 de marzo de 2021

\begin{abstract}
The development of chronic pain after surgery or persistent postoperative pain is a significant public health problem that affects between $10 \%-56 \%$ of patients undergoing surgical intervention. It produces great restrictions of mobility, limitation of daily activities, dependence on opioids, anxiety, depression, a great alteration of the quality of life and important legal and medical-economic consequences. There is a very close correlation between acute postoperative pain, and persistent postoperative pain. For this reason, pain must be treated effectively in its acute phase to reduce the incidence of chronic pain after surgery. There are risk factors that predispose to its appearance and that must be known by the anesthesiologist. In an individualized and meticulous pre-anesthetic consultation, these risk factors can be detected, as well as the presence of surgical procedures related to chronic pain, with the purpose of the therapeutic approach of the first ones if necessary, and a good planning of the anesthetic and analgesic technique, which reduces the participation of the second, in the chronicity of acute pain. The role of the anesthesiologist in the pre-anesthetic consultation is essential for the planning of preventive and multimodal analgesia that, together with other resources, should reduce the incidence of persistent postoperative pain.
\end{abstract}

Key words: Persistent postsurgical pain, chronic postsurgical pain, chronic pain, pre-anesthetic consultation, role of the anesthesiologist.

\section{RESUMEN}

El desarrollo del dolor crónico después de la cirugía o dolor posoperatorio persistente, es un problema de salud pública significativo que afecta entre el 10\%-56\% de los pacientes sometidos a una intervención quirúrgica. Produce grandes restricciones de la movilidad, limitación de las actividades de vida diaria, dependencia a los opioides, ansiedad, depresión, una gran alteración de la calidad de vida e importantes consecuencias legales y médico-económicas. Hay una correlación muy estrecha entre el dolor agudo posoperatorio, y el dolor posoperatorio persistente. Por esa razón, el dolor debe ser tratado de manera eficaz en su fase aguda para disminuir la incidencia del dolor crónico posterior a la cirugía. Existen factores de riesgo que predisponen a su aparición y que deben ser conocidos por el anestesiólogo. En una consulta pre-anestésica individualizada y minuciosa, se podrán detectar estos factores de riesgo, como también la presencia de procedimientos quirúrgicos relacionados con el dolor crónico, con la finalidad del abordaje terapéutico de los primeros de ser necesario, y una buena planificación de la técnica anestésica y analgésica, que disminuya la participación de los segundos, en la cronicidad del dolor agudo. El rol del anestesiólogo en la consulta pre-anestésica, es fundamental para la planificación de la analgesia preventiva y multimodal que junto a otros recursos deberían disminuir la incidencia del dolor postoperatorio persistente.

Palabras clave: Dolor posquirúrgico persistente, dolor crónico, consulta pre-anestésica, dolor posoperatorio persistente, rol del anestesiólogo. 


\section{Introducción}

E desarrollo del dolor crónico después de la cirugía, también llamado dolor posoperatorio persistente (DPP), o dolor crónico posoperatorio, es un problema de salud pública significativo que afecta según algunos el 10\% de todas las cirugías[1],[2],[3] y según otros entre el 20\%-56\% de los pacientes intervenidos quirúrgicamente[4]. Este dolor produce en los pacientes, grandes restricciones de la movilidad, limitación de las actividades de vida diaria, dependencia a los opioides, ansiedad, depresión, gran alteración de la calidad de vida e importantes consecuencias legales y médico-económicas[5].

Al evaluar su impacto en la población, encontramos que $66 \%$ de los enfermos lo refiere con una intensidad moderada, reportando una escala numérica verbal (ENV) de 5-7/10 y el $34 \%$ como severa con una ENV de 8-10/10. La mitad de los enfermos que lo sufren, toman fármacos para el dolor sin prescripción médica y por tiempo prolongado, lo cual representa un gran problema para los pacientes portadores de comorbilidades asociadas, agregándose un factor adicional de riesgo para posibles reacciones adversas medicamentosas (RAM). Los medicamentos que se ingieren con mayor frecuencia son: los antiinflamatorios de origen no esteroideos (AINE) 55\%, el paracetamol $43 \%$ y los opioides débiles 13\%[6].

El dolor agudo posoperatorio (DAPO) es una situación omnipresente en toda cirugía, habiendo una correlación muy estrecha entre su presencia y el DPP. Por esta razón, estamos obligados a tratarlo de manera eficaz para disminuir la presencia del dolor crónico. En condiciones normales, el dolor agudo posterior a la cirugía se resuelve en la medida que se van cicatrizando los tejidos. Sin embargo, en algunos pacientes el síntoma persiste más allá del tiempo estimado para la cicatrización, pasando a un estado de cronicidad[1].

Hay factores que predisponen a su aparición y que deben ser conocidos por el anestesiólogo. Si bien es cierto que no todos son de fácil control, hay algunos de ellos sobre los cuales se pueden realizar intervenciones para la disminución de la incidencia del DPP. Los factores que predisponen a la aparición del dolor posoperatorio crónico son[7]: genéticos, psicosociales, dolor preoperatorio, factores dependientes de la cirugía, técnica anestésica y dolor agudo postoperatorio (DAPO). (Figura 1).

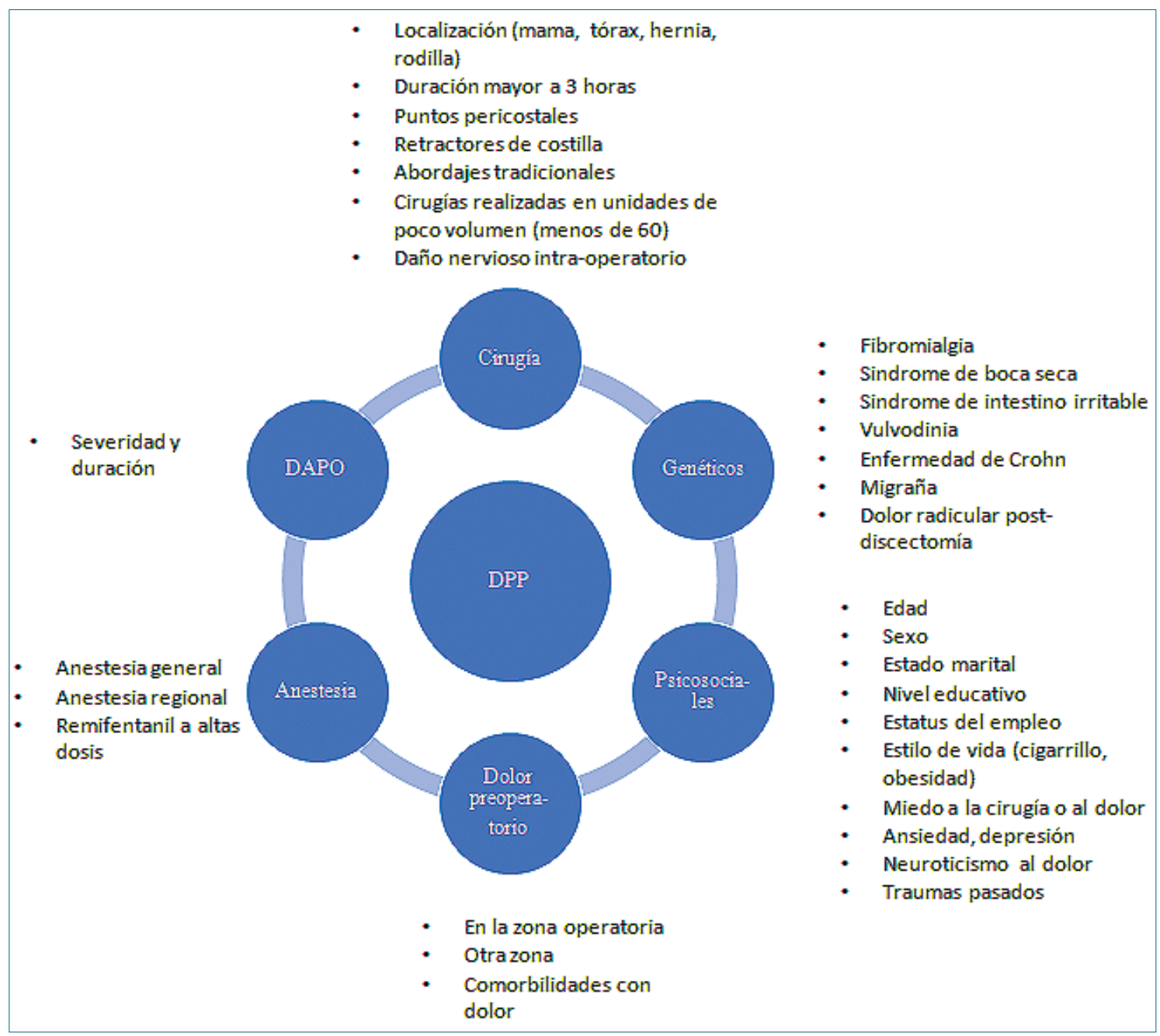

Figura 1. Factores predictores de DPP. 


\section{A. Factores genéticos}

Hay condiciones clínicas asociadas a polimorfismos genéticos, que aumentan el riesgo para el desarrollo del dolor crónico. Si el enfermo ya tiene un diagnóstico previo, ellas pudieran ser identificadas en la consulta preanestésica mediante un interrogatorio sencillo. El objetivo de esto, es buscar la orientación y tratamientos adecuados para disminuir la influencia de estos factores en la cronicidad del DAPO. Entre esas condiciones asociadas a polimorfismos genéticos mencionaremos: la migraña, el síndrome de intestino irritable, la fibromialgia, el síndrome de boca quemante, la vulvodinia, la enfermedad de Crohn, el dolor radicular posdisectomía y el síndrome de Raynaud[8] (Tabla 1).

\section{B. Factores psicosociales}

Son predictores significativos de dolor crónico posterior a la cirugía. Se incluyen entre ellos: el miedo a la operación, el miedo al dolor, la ansiedad, la depresión, el neuroticismo y los traumas pasados[7].

También hay predictores somáticos importantes que anticipan un resultado desfavorable para el desarrollo del dolor crónico posterior a una intervención quirúrgica. Ellos son la duración de la misma y los niveles altos de DAPO. Los pacientes que refieren altas escalas de dolor. hasta cuatro días después de la operación y los que son sometidos a una cirugía mayor de 3 $\mathrm{h}$ de duración, tienen mayor riesgo de aumento del dolor, aumento de las limitaciones funcionales, recuperación global deficiente y más baja calidad de vida seis meses después de la operación. Las variables psicológicas más importantes que influyen en esos resultados son el miedo preoperatorio a la cirugía y el optimismo. Este último, se asocia con una mejor recuperación y disminución de la discapacidad física, el bienestar y una mayor calidad de vida[9],[10]. Todos estos predictores psicosociales pueden ser detectados en el preoperatorio durante la consulta preanestésica, ya sea para abordarlos anticipadamente o reforzarlos como en el caso del optimismo.

\section{Dolor preoperatorio}

La presencia de dolor preoperatorio, no necesariamente en la zona a ser intervenida, es un factor de riesgo importante a ser considerado en el desarrollo de dolor crónico poscirugía [8],[35],[54],[55],[56],[57],[58].

La identificación oportuna de pacientes con dolor previo a la cirugía ayudará a mejorar la estratificación del riesgo y a la planificación de las intervenciones terapéuticas, que ayudarán a disminuir la posibilidad de desarrollar dolor crónico posquirúrgico. La asociación de dolor preoperatorio, la tendencia al catastrofismo al dolor y la ansiedad, son predictores importantes de la intensidad del DAPO en las primeras $24 \mathrm{~h}$ posteriores a la cirugía[11]. Se cree que este riesgo surge por la sensibilización de los nociceptores dentro del campo quirúrgico y cambios estructurales en el sistema nervioso central (SNC), lo cual predispone a la sensibilización al dolor debido a los estímulos dolorosos preexistentes[12]. Como ejemplo, se mencionan las mastectomías y las amputaciones de miembros, donde el dolor preoperatorio aumenta el riesgo de dolor fantasma en el posoperatorio[13].

\section{Factores dependientes de la cirugía}

Son factores muy importantes para el desarrollo del dolor crónico posquirúrgico, para los cuales los anestesiólogos poco podemos ofrecer para lograr su disminución, por depender exclusivamente del cirujano. Entre ellos se mencionan[7]: la localización de la cirugía, las intervenciones quirúrgicas de más de tres horas de duración, el uso de puntos peri costales en la cirugía torácica, el uso de retractores de costilla, los abordajes tradicionales versus los laparoscópicos, las cirugías realizadas en unidades quirúrgicas de poco volumen (menos de 60/mes) y el daño nervioso intraoperatorio.

Entre las cirugías más relacionadas con el DPP se mencionan: la amputación de miembros (30\%-85\%), la artroplastia de rodilla (13\%-44\%), la cesárea (55\%-86\%), la reparación de hernia inguinal (5\%-63\%), la laminectomía y fusión espinal

\begin{tabular}{lll}
\hline & Tabla 1. Condiciones clínicas asociadas a polimorfismos genéticos \\
\hline Enfermedad & Gen relacionado & Codificador de: \\
Fibromialgia & 5 -HTTLPR & Transportados de serotonina \\
Sínd. de boca ardiente & 5 -HTTLPR & Transportados de serotonina \\
Sínd. intestino irritable & 5 -HTTLPR & Transportados de serotonina \\
Fibromialgia & 5 -HTTLPR & Transportados de serotonina \\
Sínd. de boca seca & L1B & IL-1b \\
Vulvodinia & MC1R & Receptor de melanocortina 1 \\
Enf. de Crohn & IL23R & Subunidad del receptor para IL-23 \\
Dolor radicular persistente posdiscectomía & GCH1 & GTP ciclohidrolasa \\
Migraña & COMT & Catecol-O-metilo transferasa, enzima que inactiva la \\
Dolor crónico & & dopamina, epinefrina y norepinefrina en el SNC \\
\hline
\end{tabular}

Sínd.(Síndrome). Enf. (Enfermedad). 
Tabla 2. Recursos y procedimientos ahorradores de opioides[16],[17]

\begin{tabular}{|c|c|c|c|}
\hline Intervención & Tipo/Dosis & Efectos & RAM \\
\hline Técnicas de anestesia local & $\begin{array}{l}\text { Catéteres en plexos, anestesia } \\
\text { epidural, catéteres en la herida, } \\
\text { infiltraciones, bloqueos interfasciales }\end{array}$ & $\begin{array}{l}\downarrow 50 \% \text { la necesidad de opioides } \\
\text { Útil en dependientes de opioides }\end{array}$ & Hipotensión \\
\hline $\begin{array}{l}\text { Acetaminofeno } \\
\text { (Paracetamol) }\end{array}$ & $\begin{array}{l}1 \mathrm{~g} \times 4 \text { en adultos } \\
1 \mathrm{~g} \times 3 \text { en adultos }\end{array}$ & $\begin{array}{l}\downarrow \text { Necesidad opioide } 20 \%-30 \% \\
\text { Sinergismo con AlNEs y Coxibs }\end{array}$ & Insuficiencia hepática \\
\hline AINE, Coxibs & $\begin{array}{l}\text { Ketorolaco } 30 \mathrm{mg} \\
\text { Celecoxib } 400 \mathrm{mg}\end{array}$ & $\begin{array}{l}\downarrow \text { Uso de opioides } \\
\text { Sinergismo con paracetamol }\end{array}$ & $\begin{array}{l}\text { Efectos renales, hepáticos, } \\
\text { cardiovasculares y gastrointestinales }\end{array}$ \\
\hline Gabapentina & 100-300 VO. mg OD & Dolor neuropático & Riesgo de sedación postoperatoria \\
\hline Pregabalina & 50-75 VO. OD & Inciertos en ahorro de opioides & \\
\hline Ketamina & $\begin{array}{l}\text { Bolo de } 0,25-0,5 \mathrm{mg} / \mathrm{kg} \\
\text { Infusión: } 0,25-0,5 \mathrm{mg} / \mathrm{kg} / \mathrm{h}\end{array}$ & $\begin{array}{l}\downarrow \text { Necesidad de opioides } \\
\downarrow \text { HIO y náuseas y vómitos PO }\end{array}$ & $\begin{array}{l}\text { Alucinaciones, agitación, visión doble } \\
\text { ansiedad, disforia y euforia }\end{array}$ \\
\hline Magnesio & Infusión: 40-50 mg/kg & $\downarrow$ Uso de opioides y $\downarrow$ dolor PO & $\begin{array}{l}\text { Bradicardia } \\
\text { Prolonga la acción de los RMND }\end{array}$ \\
\hline Lidocaina & $\begin{array}{l}\text { Bolo: } 1-2 \mathrm{mg} / \mathrm{kg} \\
\text { Infusión: } 1,5 \mathrm{mg} / \mathrm{kg} / \mathrm{h}\end{array}$ & $\begin{array}{l}\downarrow \text { Dolor y consumo de opioides } \\
\text { Properistáltico gastrointestinal }\end{array}$ & $\begin{array}{l}\text { Vigilar toxicidad cardíaca, contractilidad } \\
\text { miocárdica y convulsiones }\end{array}$ \\
\hline Clonidina & $\begin{array}{l}\text { Preanestesia: } 75 \mu \mathrm{g} \text { VO. } \\
\text { Infusión: } 0,1-0,3 \mu \mathrm{g} / \mathrm{kg} / \mathrm{h}\end{array}$ & $\begin{array}{l}\downarrow \text { Abstinencia } \\
\text { Potencia efectos de la morfina }\end{array}$ & Hipotensión, bradicardia y sedación \\
\hline Dexmedetomidina & Infusión en PO: 0,3-0,8 $\mu \mathrm{g} / \mathrm{kg} / \mathrm{h}$ & $\downarrow$ Uso de opioides en el PO & Hipotensión, bradicardia y sedación \\
\hline Dexametasona & Preoperatorio $8 \mathrm{mg}$ & $\downarrow$ DAPO y necesidades opioides & Vigilar hiperglicemia \\
\hline
\end{tabular}

RAM: reacciones adversas medicamentosas; AINE: antiinflamatorios no esteroideos; Coxibs: inhibidores de la ciclooxigenasa; HIO: hiperalgesia inducida por opioides; VO: vía oral; OD: orden diaria; PO: posoperatorio; DAPO: dolor agudo postoperatorio; RMND: relajantes musculares no depolarizantes.

(10\%-40\%), la mastectomía (11\%-57\%), la toracotomía (5\%$65 \%)$, el bypass aortocoronario (30\%-50\%), el reemplazo de cadera $(27 \%)$, la craneotomía $(0 \%-65 \%)$ y la colecistectomía (3\%-50\%)[37].

Como contribución en este punto, los anestesiólogos estamos obligados a brindar al paciente las mejores condiciones de relajación muscular para que el equipo quirúrgico haga su trabajo lo mejor y más rápido posible, evitando así cirugías prolongadas, que parecen ser un factor relacionado con el DAPO y el DPP[7].

\section{E. Técnica anestésica}

Es un tema controvertido. Se dice que los anestésicos halogenados contribuyen a la inflamación neurogénica, al activar canales iónicos periféricos que transmiten señales nociceptivas, que pueden contribuir al desarrollo del dolor crónico[14]. También se comenta de la hiperalgesia inducida por los opioides como un factor predictor del DPP, razón por la cual deberíamos utilizar menos opioides en el perioperatorio y apoyarnos con mayor frecuencia en otros recursos farmacológicos y procedimientos. Entre ellos: la analgesia multimodal[38],[39], la dexmedetomidina[40],[41], la ketamina[42], la lidocaína intravenosa[43], el sulfato de magnesio[44], la gabapentina[45],[46], la dexametasona[47], el paracetamol[48], los antiinflamatorios no esteroideos (AINE)[49], los inhibidores de la ciclooxigenasa-2 (COX-2)[50], la infiltración de anestésicos locales (bupivacaina liposomal)[51],[52] y los bloqueos en el neuroeje[15],[16],[17] (Tabla 2).

En relación con el uso del remifentanilo en el trans-operatorio, se comenta que el uso de dosis altas $(0,4 \mu \mathrm{g} / \mathrm{kg} / \mathrm{min}$ vs 0,05 $\mu \mathrm{g} / \mathrm{kg} / \mathrm{min})$, parece ser un factor predictivo para el desarrollo del DAPO y la hiperalgesia inducida por opioides (HIO), factores que pueden ser desencadenantes del DPP[18].

\section{F. Dolor agudo posoperatorio (DAPO)}

El dolor posoperatorio agudo es un factor predictivo importante para el dolor crónico posterior a la cirugía, fundamentalmente en algunas intervenciones quirúrgicas como: la reparación de hernia inguinal, la cirugía torácica, la cesárea, el reemplazo de rodilla, la artroplastia total de cadera, la cirugía de cáncer de mama, y la amputación de extremidades[13],[19],[20],[21]. Existe una fuerte correlación entre el DAPO y el dolor crónico poscirugía, particularmente en las toracotomías debido a una extensa lesión nerviosa en el sitio quirúrgico. Así que, el DAPO en el posoperatorio inmediato, es un objetivo clave a solucionar, en los esfuerzos para la disminución del riesgo de dolor crónico posterior a la cirugía[13],[20],[21],[22],[56],[57],[58].

\section{Estrategias para la prevención del DPP a ser usadas por el anestesiólogo (Tabla 3)}

Es muy importante que antes de la intervención quirúrgica, el anestesiólogo tenga presente los predictores de riesgo de DPP, así como también las diferentes cirugías que con mayor frecuencia son determinantes para el desarrollo de dolor crónico. Sólo de esa manera, se podrán detectar precozmente los pacientes que tienen más riesgo de presentar el DPP, para su abordaje, orientación y tratamientos oportunos que minimicen la enfermedad. En este sentido, una buena consulta preanes- 
Tabla 3. Estrategias a ser usadas por el anestesiólogo en el perioperatorio, para disminuir el dolor posoperatorio persistente (DPP)

Preoperatorio

1) Consulta PA individualizada y bien planiicada

2) Detección de factores psicológicos

3) Detección de polimorfismos genéticos

4) Detección de cirugías asociadas con DAPO

5) Presencia de dolor zona operatoria y fuera de ella

6) Abordaje terapético, si aplica (gabapentoides, AINE)

7) Planificar la técnica anestésica y analgésica

8) Informar factores de riesgo al cirujano

9) Transmitir seguridad y confianza

10) Buscar optimismo en el paciente

\section{Intraoperatorio}

1) En cirugía mayor usar ketamina. Bolo: 0,5 $\mathrm{mg} / \mathrm{kg}+\mathrm{IC}: 10 \mathrm{mcg} / \mathrm{kg} / \mathrm{min}$

2) En cirugía abdominal abierta, Lidocaina bolo $1-1,5 \mathrm{mg} / \mathrm{kg}+\mathrm{IC}: 2 \mathrm{mg} / \mathrm{kg} / \mathrm{h}$

3) Dosis bajas de remifentamil: $0,05 \mathrm{mcg} / \mathrm{kg} / \mathrm{min}$

4) Relajacion muscular adecuada

5) Infiltración subcutánea y subfascial con anestésico local de duración prolongada

6) De preferencia anestesia regional y/o en el neuroeje

7) Técnica ahorrativa de opioides para disminuir la $\mathrm{HIO}$

8) Continuar la analgesia preventiva y multimodal

9) Preparar el paciente para una analgesia posoperatoria eficaz

\section{Posoperatorio}

1) Continuar la analgesia preventiva y multimodal 2) Si aplica, Paracetamol y/o AINE

3) Planificar la medicación posoperatoria de egreso

4) Referir a la clínica de dolor transicional (si se dispone de ella) o a los servicios de dolor agudo

PA: preanestésica; DAPO: dolor agudo posoperatorio; IC: infusión contínua; AINE: antiinflamatorios de origen no esteroideos; HIO: hiperalgesia inducida por opioides.

tésica es fundamental, permitiéndole al anestesiólogo realizar planes analgésicos individualizados y el uso de los recursos farmacológicos y procedimentales que permitirán disminuir el riesgo de dolor crónico después de la cirugía.

Las estrategias usadas para la prevención del DPP son:

\section{Analgesia preventiva}

Este recurso es un término que se refiere al tratamiento que se comienza antes de la cirugía, y está destinado a prevenir la sensibilización periférica y central del sistema a nervioso, causada por la incisión quirúrgica y otras respuestas inflamatorias a la cirugía. Su objetivo es disminuir la excitación de las fibras aferentes primarias y el proceso inflamatorio, que son elementos fundamentales en el desarrollo del DAPO severo y el dolor crónico posterior a la cirugía[7],[23],[24],[58]. Si bien es cierto que al día de hoy no hay una evidencia fuerte para su recomendación, esto no la excluye de su uso con la finalidad de disminuir la intensidad del DAPO y la aparición del DPP[58]. Se mencionan como métodos multimodales eficaces, la analgesia preventiva con antiinflamatorios no esteroideos (AINE) selectivos y no selectivos de la ciclooxigenasa 2 (COX2), los gabapentinoides, el acetaminofén y la infiltración de anestésicos locales[52]. La analgesia preventiva puede mejorar la satisfacción del paciente y la calidad de vida al reducir el DAPO y el consumo de opioides[53].

\section{Analgesia multimodal}

Se refiere al uso de técnicas analgésicas en diferentes formas, dirigidas a los distintos niveles de la nocicepción (percepción del estímulo doloroso), con el objetivo de prevenir y tratar el dolor crónico posterior a la cirugía. Estas técnicas tienen como finalidad, la disminución de la excitabilidad de las neuronas involucradas en el proceso nociceptivo, tanto a nivel cen- tral como periférico, y se logra con diferentes recursos, entre ellos: la anestesia neuroaxial, regional y local[7],[23] y también con el empleo de fármacos como la ketamina, el esmolol, la lidocaína y la dexmedetomidina, que disminuyen el consumo posoperatorio de opioides, y reducen el riesgo de desarrollo de dolor crónico posquirúrgico[27],[28]. Fármacos como la ketamina, el oxido nitroso y la metadona, inactivan los receptores $\mathrm{N}$-metil-D-aspartato (NMDA) siendo también beneficiosos en la prevención del dolor crónico posoperatorio. Igualmente son útiles bajo el mismo concepto multimodal, fármacos como la gabapentina y la pregabalina, que usados en el período perioperatorio, parecen disminuir la sensibilización producida por la cirugía disminuyendo el DAPO y el dolor crónico posquirúrgico en algunas intervenciones específicas, como la reparación de hernia inguinal y la artroplastia de rodilla[29],[30]. No debemos dejar de mencionar bajo el mismo enfoque, el uso de los anestésicos locales que pueden ayudar aún más en la prevención del DPP. Su uso en el campo quirúrgico durante el intraoperatorio disminuye la gravedad del DAPO y la posibilidad de desarrollar dolor crónico posterior a la cirugía, sobre todo si se usa junto con un AINE como el ketorolaco[31],[32].

La anestesia regional y neuraxial puede ser de gran utilidad como herramientas preventivas del dolor crónico posoperatorio, sobre todo en cirugías como la toracotomía, la histerectomía, la cesárea y la cirugía mamaria[33],[34].

Los procedimientos multimodales deben ser usados en el preoperatorio, intraoperatorio y posoperatorio, con la finalidad de disminuir la nocicepción, la hiperalgesia primaria y secundaria y el desarrollo del DPP[17],[23].

Debemos tener presente que la severidad del DAPO es un elemento clave para el desarrollo del DPP, resultando la técnica anestésica utilizada una pieza fundamental para su aparición y evolución. Por lo tanto, la planificación del abordaje terapéuti- 
co, debe englobar las tres etapas del proceso quirúrgico, como son: el preoperatorio, intraoperatorio y posoperatorio[35].

\section{A. Preoperatorio}

La prevención del DPP debe comenzar en el preoperatorio, con la realización de una consulta preanestésica bien planificada e individualizada, con objetivos bien definidos, como lo son la identificación y abordaje de los factores de riesgo y dotar al paciente de estrategias para la prevención del dolor crónico[59]. En la consulta se deben considerar entre otras preguntas al paciente, las relacionadas con la existencia de dolor crónico y el uso regular de analgésicos y coanalgésicos[59]. De estar indicados, se pueden prescribir algunos fármacos antes de la cirugía, como los anticonvulsivantes y los AINE inhibidores selectivos de la COX2, sin olvidar su contraindicación en la cardiopatía isquémica y en los pacientes programados para bypass coronario[36].

De ser necesario, se puede optar en el preoperatorio de recursos de la medicina complementaria o alternativa, para disminuir la ansiedad preoperatoria y la intensidad de dolor preoperatorio. Es el caso de la acupuntura[60] y la músicoterapia[61],[62]

¿Que debemos hacer en la consulta preanestésica?:

1. Detección y abordaje terapéutico de ser posible, de factores de riesgo psicológico (neuroticismo, ansiedad, introversión, dolor preoperatorio, miedo a la cirugía, miedo al dolor, traumas pasados, etc.)[7].

2. Detección y abordaje terapéutico de ser posible, de comorbilidades asociadas a polimorfismos genéticos, que puedan incidir directa o indirectamente en el desarrollo del DPP (migraña, síndrome de intestino irritable, fibromialgia, síndrome de boca quemante, vulvodinia, enfermedad de Crohn, dolor radicular posdisectomía, síndrome de Reyunad)[8].

3. Detección de cirugías relacionadas con mayor incidencia de DAPO y DPP (amputaciones, toracotomías, mastectomías, bypass coronario, herniorrafias inguinales, cirugía torácica, cesárea, reemplazo de rodilla, artroplastia total de cadera, cirugía de cáncer de mama y amputación de extremidades[13],[19],[20],[21].

4. Buscar la presencia de dolor preoperatorio, en la zona a ser intervenida y fuera de ella[8],[11].

5. Abordaje terapéutico y plan de tratamiento de los factores mencionados, de ser posible. Medicar si está indicado con anticonvulsivantes (gabapentina y pregabalina) y AINE[25],[26],[29],[30].

6. Planificar la técnica anestésica de manera individualizada, considerando los factores mencionados anteriormente y la cirugía a realizar. Se deben incluir la analgesia preventiva y la multimodal, considerando el preoperatorio, intraoperatorio y posoperatorio[15],[16],[17],[35].

7. Planificar la técnica analgésica considerando la cirugía a realizar y tomando en cuenta las posibilidades de desarrollar DPP.

8. Si se detectan factores de riesgo en la consulta preanestésica, se debe alertar al cirujano de la posibilidad de desarrollo de DPP.

9. Referencia oportuna a otros médicos y servicios de ser necesario, antes de la cirugía.

10. Dar el máximo de confianza y seguridad a los enfermos con tendencia al neuroticismo[9],[10].
11. Buscar siempre optimismo en el preoperatorio de los pacientes. Esta conducta se asocia a una mejor recuperación, disminución de la discapacidad física, el bienestar y una mayor calidad de vida[9],[10].

\section{B. Intraoperatorio}

Durante el intraoperatorio, se pueden adoptar algunas conductas y técnicas para disminuir la incidencia del DPP, sobre todo al detectarse factores de riesgo en la consulta preanestésica. Entre ellas:

1. Si no hay contraindicación se puede usar en cirugía mayor, la ketamina intravenosa a una dosis de impregnación es 0,5 mg/kg seguido de una infusión de $10 \mu \mathrm{g} / \mathrm{kg} /$ $\min [16],[17],[18]$.

2. En la cirugía abdominal abierta y laparoscópica se puede usar lidocaína IV, a la dosis bolo de 1-1,5 mg/kg seguido de una infusión intraoperatoria de $2 \mathrm{mg} / \mathrm{k} / \mathrm{h}[16],[17],[27]$.

3. Si se va a utilizar el remifentanilo como opioide, se recomienda usar dosis bajas $(0,05 \mu \mathrm{g} / \mathrm{kg} / \mathrm{min})[16],[17],[18]$.

4. Adecuada relajación muscular que facilite el trabajo del equipo quirúrgico.

5. Infiltración subcutánea y subfascial antes de finalizar la cirugía, preferentemente con anestésicos locales de duración prolongada (cesáreas, laparotomías, hemorroidectomías).

6. Infiltración intra-articular de anestésicos locales u opioides.

7. Usar técnicas en el neuroeje o de anestesia/analgesia regional[33],[34].

8. Usar técnicas anestésicas ahorrativas de opioides para evitar la hiperalgesia inducida por opioides (HIO)[16],[17],[18] (Tabla 2).

9. Continuar la analgesia preventiva y multimodal iniciada en el preoperatorio.

10. Preparar al paciente para una analgesia posoperatoria eficaz.

\section{Posoperatorio}

a. Continuar con la analgesia preventiva y multimodal.

b. Paracetamol y/o AINE: solos o en combinación, están recomendados para la reducción de la dosis de los opioi$\operatorname{des}[16],[17]$.

c. Planificar la medicación analgésica de egreso e informarlo al cirujano.

d. Lo deseable sería hacer un seguimiento del paciente en el posoperatorio de por lo menos seis meses, a través de las clínicas de dolor transicional o clínicas de dolor agudo, diseñadas para para la vigilancia y control de estos enfermos. Se busca detectar y tratar, factores de riesgo que nos anticipen el dolor crónico posoperatorio[1],[17],[37]. También nos permiten hacer un buen seguimiento del uso y destete de los opioides, si fueron prescritos en la medicación de egreso. Estas unidades son modelos que abordan de manera integral el problema del dolor crónico después de la cirugía sirviendo de intermediarias entre el manejo del DAPO en la sala de hospitalización y el manejo del dolor crónico ambulatorio[1],[17],[37].

\section{Conclusiones}

El rol del médico anestesiólogo en la prevención del dolor 
crónico es fundamental. Su visión holística del paciente quirúrgico, pudiera disminuir la incidencia del DPP, debido a que tiene una participación activa en las tres etapas del acto quirúrgico, donde pueden realizar acciones que logren disminuir la posibilidad del dolor crónico posterior a la cirugía. Comenzando con la consulta pre anestésica en el preoperatorio, donde puede detectar factores y procedimientos quirúrgicos predictores de riesgo, continuando con el intraoperatorio donde puede escoger la técnica anestésica y los procedimientos que disminuyan la posibilidad de estimulación y sensibilización de los nociceptores y finalizando con el posoperatorio, donde puede tener un papel relevante para lograr una menor duración y severidad del DAPO, colaborar en la formulación de una medicación de egreso adecuada a la intensidad y características del dolor al momento del egreso, así como también realizar seguimiento de los pacientes operados, siempre pensando en la detección y tratamiento de factores de riesgo de DPP. Como parte de las estrategias para disminuir el dolor crónico siempre debe pensar en la analgesia preventiva y la analgesia multimodal.

\section{Referencias}

1. Katz J, Weinrib A, Fashler SR, Katznelzon R, Shah BR, Ladak SS, et al. The Toronto General Hospital Transitional Pain Service: development and implementation of a multidisciplinary program to prevent chronic postsurgical pain. J Pain Res. 2015 Oct;8:695702. https://doi.org/10.2147/JPR.S91924 PMID:26508886

2. Macrae WA. Chronic post-surgical pain: 10 years on. Br J Anaesth. 2008 Jul;101(1):77-86. https://doi.org/10.1093/bja/ aen099 PMID:18434337

3. Richebé P, Capdevila X, Rivat C. Persistent Postsurgical Pain: Pathophysiology and Preventative Pharmacologic Considerations. Anesthesiology. 2018 Sep;129(3):590-607. https://doi. org/10.1097/ALN.0000000000002238 PMID:29738328

4. Montes A, Roca G, Sabate S, Lao JI, Navarro A, Cantillo J, et al.; GENDOLCAT Study Group. Genetic and clinical factors associated with chronic postsurgical pain after hernia repair, hysterectomy, and thoracotomy: A two-year multicenter cohort study. Anesthesiology. 2015 May;122(5):1123-41. https://doi.org/10.1097/ ALN.0000000000000611 PMID:25985024

5. Washington DC. US Department of Health and Human Services. National Institutes of Health; 2016.

6. Breivik H, Collett B, Ventafridda V, Cohen R, Gallacher D. Survey of chronic pain in Europe: prevalence, impact on daily life, and treatment. Eur J Pain. 2006 May; 10(4):287-333. https://doi. org/10.1016/j.ejpain.2005.06.009 PMID:16095934

7. Fregoso G, Wang A, Tseng K, Wang J. Transition from Acute to Chronic Pain: Evaluating Risk for Chronic Postsurgical Pain. Pain Physician. 2019 Sep;22(5):479-88. PMID:31561647

8. Katz J, Seltzer Z. Transition from acute to chronic postsurgical pain: risk factors and protective factors. Expert Rev Neurother. 2009 May;9(5):723-44. https://doi.org/10.1586/ern.09.20 PMID:19402781

9. Peters ML, Sommer M, de Rijke JM, Kessels F, Heineman E, Patijn J, et al. Somatic and psychologic predictors of longterm unfavorable outcome after surgical intervention. Ann Surg. 2007 Mar;245(3):487-94. https://doi.org/10.1097/01. sla.0000245495.79781.65 PMID:17435557

10. Granot M, Ferber SG. The roles of pain catastrophizing and anxiety in the prediction of postoperative pain intensity: a prospective study. Clin J Pain. 2005 Sep-Oct;21(5):439-45. https:// doi.org/10.1097/01.ajp.0000135236.12705.2d PMID:16093750

11. Khalil H, Shajrawi A, Dweik G, Zaghmouri A, Henker R. The impact of preoperative pain related psychological factors on pain intensity post-surgery in Jordan. J H P; 2020. https://doi. org/10.1177/1359105320937067.

12. Dubner R, Ruda MA. Activity-dependent neuronal plasticity following tissue injury and inflammation. Trends Neurosci. 1992 Mar;15(3):96-103. https://doi.org/10.1016/01662236(92)90019-5 PMID:1373925

13. Nikolajsen L, IIkjaer S, Krøner K. Chris- tensen JH, Jensen TS. The influence of pre- amputation pain on post-amputation stump and phantom pain. Pain. 1997;72:393-405. https://doi.org/10.1016/ S0304-3959(97)00061-4 PMID:9313280

14. Matta JA, Cornett PM, Miyares RL, Abe K, Sahibzada N, Ahern GP. General anesthetics activate a nociceptive ion channel to enhance pain and inflammation. Proc Natl Acad Sci USA. 2008 Jun;105(25):8784-9. https://doi.org/10.1073/pnas.0711038105 PMID: 18574153

15. Thota RS, Ramkiran S, Garg R, Goswami J, Baxi V, Thomas M. Opioid free onco-anesthesia: Is it time to convict opioids? A systematic review of literature. http://www.joacp.org on Tuesday, November 17, 2020, IP: 190.73.87.7]

16. Baboli KM, Liu H, Poggio JL. Opioid-free postoperative analgesia: is it feasible? Curr Probl Surg. 2020 Jul;57(7):100794. https://doi. org/10.1016/j.cpsurg.2020.100794 PMID:32546291

17. Miclescu A. Chronic pain patient and anaesthesia. Rom J Anaesth Intensive Care. 2019 Apr;26(1):59-66. https://doi.org/10.2478/ rjaic-2019-0009 PMID:31111097

18. Lavand'homme P, Steyaert A. Opioid-free anesthesia opioid side effects: tolerance and hyperalgesia. Best Pract Res Clin Anaesthesiol. 2017 Dec;31(4):487-98. https://doi.org/10.1016/j. bpa.2017.05.003 PMID:29739537

19. Liu SS, Buvanendran A, Rathmell JP, Sawhney M, Bae JJ, Moric $M$, et al. A cross-sectional survey on prevalence and risk factors for persistent postsurgical pain 1 year after total hip and knee replacement. Reg Anesth Pain Med. 2012 Jul-Aug;37(4):415-22. https://doi.org/10.1097/AAP.0b013e318251b688 PMID:22660483

20. Poleshuck EL, Katz J, Andrus CH, Hogan LA, Jung BF, Kulick DI, et al. Risk factors for chronic pain following breast cancer surgery: a prospective study. J Pain. 2006 Sep;7(9):626-34. https://doi. org/10.1016/j.jpain.2006.02.007 PMID:16942948

21. Kehlet $H$, Jensen $T S$, Woolf $C J$. Persistent postsurgical pain: risk factors and prevention. Lancet. 2006 May;367(9522):161825. https://doi.org/10.1016/S0140-6736(06)68700-X PMID:16698416

22. Buvanendran A, Della Valle CJ, Kroin JS, Shah M, Moric M, Tuman $\mathrm{KJ}$, et al. Acute postoperative pain is an independent predictor of chronic postsurgical pain following total knee arthroplasty at 6 months: a prospective cohort study. Reg Anesth Pain Med. 2019 Mar;44(3):rapm-2018-100036. https://doi.org/10.1136/ rapm-2018-100036 PMID:30770420

23. Fiezerfan A, Sheh G. Transition from acute to chronic pain. Contin Educ Anaesth Crit Care Pain. 2015;15(2):98-102. https://doi. org/10.1093/bjaceaccp/mku044.

24. McGreevy K, Bottros MM, Raja SN. Preventing chronic pain following acute pain: risk factors, preventive strategies, and their efficacy. Eur J Pain Suppl. 2011 Nov;5(2):365-72. https://doi. 
org/10.1016/j.eujps.2011.08.013 PMID:22102847

25. Michaloliakou C, Chung F, Sharma S. Preoperative multimodal analgesia facilitates recovery after ambulatory laparoscopic cholecystectomy. Anesth Analg. 1996 Jan;82(1):44-51. PMID:8712425

26. Kien NT, Geiger P, Van Chuong H, Cuong NM, Van Dinh N, Pho $D C$, et al. Preemptive analgesia after lumbar spine surgery by pregabalin and celecoxib: a prospective study. Drug Des Devel Ther. 2019 Jul;13:2145-52. https://doi.org/10.2147/DDDT.S202410 PMID:31308627

27. Dunn LK, Durieux ME. Perioperative use of intravenous lidocaine. Anesthesiology. 2017 Apr;126(4):729-37. https://doi. org/10.1097/ALN.0000000000001527 PMID:28114177

28. Habib AS, Gan TJ. Role of analgesic adjuncts in postoperative pain management. Anesthesiol Clin North America. 2005 Mar;23(1):85-107. https://doi.org/10.1016/j.atc.2004.11.007 PMID:15763413

29. Clarke H, Bonin RP, Orser BA, Englesakis M, Wijeysundera DN, Katz J. The prevention of chronic postsurgical pain using gabapentin and pregabalin: a combined systematic review and metaanalysis. Anesth Analg. 2012 Aug;115(2):428-42. https://doi. org/10.1213/ANE.0b013e318249d36e PMID:22415535

30. Sen H, Sizlan A, Yanarates $O$, Senol MG, Inangil G, Sücüllü I, et al. The effects of gabapentin on acute and chronic pain after inguinal herniorrhaphy. Eur J Anaesthesiol. 2009 Sep;26(9):772-6. https://doi.org/10.1097/EJA.0b013e32832ad2fa PMID:19424073

31. Kurmann A, Fischer $H$, Dell-Kuster $S$, Rosenthal R, Audigé L, Schüpfer $G$, et al. Effect of intraoperative infiltration with local anesthesia on the development of chronic pain after inguinal hernia repair: a randomized, triple-blinded, placebo-controlled trial. Surgery. 2015 Jan;157(1):144-54. https://doi.org/10.1016/j. surg.2014.07.008 PMID:25482469

32. Bugada D, Lavand'homme P, Ambrosoli AL, Cappelleri G, Saccani Jotti GM, Meschi T, et al. Effect of Preoperative Inflammatory Status and Comorbidities on Pain Resolution and Persistent Postsurgical Pain after Inguinal Hernia Repair. Mediators Inflamm. 2016;2016:5830347. https://doi.org/10.1155/2016/5830347 PMID:27051077

33. McCartney CJ, Brull R, Chan VW, Katz J, Abbas S, Graham B, et al. Early but no long-term benefit of regional compared with general anesthesia for ambulatory hand surgery. Anesthesiology. 2004 Aug;101(2):461-7. https://doi.org/10.1097/00000542200408000-00028 PMID:15277930

34. Weinstein EJ, Levene JL, Cohen MS, Andreae DA, Chao JY, Johnson $\mathrm{M}$, et al. Local anaesthetics and regional anaesthesia versus conventional analgesia for preventing persistent postoperative pain in adults and children. Cochrane Database Syst Rev. 2018 Apr;4:CD007105. PMID:29694674

35. Ribera H, Esteve N, Garrido JP. La transición de dolor agudo postoperatorio a crónico: ¿qué sabemos? Rev Soc Esp Dolor. 2012;19(4):197-208.

36. Ramos-Alaniz A, Guajardo-Rosas J, Chejne-Gómez F, JuárezLemus A, Ayón-Villanueva H. Mecanismos para prevenir dolor agudo a crónico. Rev. Mex. Anestesiol. 2018;41(1):\$44-7.

37. Glare P, Aubrey KR, Myles PS. Transition from acute to chronic pain after surgery. Lancet. 2019 Apr;393(10180):153746. https://doi.org/10.1016/S0140-6736(19)30352-6 PMID:30983589

38. Kehlet H, Dahl JB. The value of "multimodal" or "balanced analgesia" in postoperative pain treatment. Anesth Analg. 1993 Nov;77(5):1048-56. https://doi.org/10.1213/00000539-
199311000-00030 PMID:8105724

39. Elvir-Lazo OL, White PF. The role of multimodal analgesia in pain management after ambulatory surgery. Curr Opin Anaesthesiol. 2010 Dec;23(6):697-703. https://doi.org/10.1097/ ACO.0b013e32833fad0a PMID:20847690

40. Lin TF, Yeh YC, Lin FS, Wang YP, Lin CJ, Sun WZ, et al. Effect of combining dexmedetomidine and morphine for intravenous patient-controlled analgesia. Br J Anaesth. 2009 Jan;102(1):11722. https://doi.org/10.1093/bja/aen320 PMID:18987053

41. Salman N, Uzun S, Coskun F, Salman MA, Salman AE, Aypar $U$. Dexmedetomidine as a substitute for remifentanil in ambulatory gynecologic laparoscopic surgery. Saudi Med J. 2009 Jan;30(1):77-81. PMID:19139778

42. Bell RF, Kalso EA. Ketamine for pain management. Pain Rep. 2018 Aug;3(5):e674. https://doi.org/10.1097/ PR9.0000000000000674 PMID:30534625

43. Eipe N, Gupta S, Penning J. Intravenous lidocaine for acute pain: an evidence-based clinical update. BJA Educ. 2016;16(9):292-8. https://doi.org/10.1093/bjaed/mkw008.

44. Lysakowski C, Dumont L, Czarnetzki C, Tramèr MR. Magnesium as an adjuvant to postoperative analgesia: a systematic review of randomized trials. Anesth Analg. 2007 Jun;104(6):1532-9. https://doi.org/10.1213/01.ane.0000261250.59984.cd PMID:17513654

45. Lunn TH, Husted H, Laursen MB, Hansen LT, Kehlet H. Analgesic and sedative effects of perioperative gabapentin in total knee arthroplasty: a randomized, double-blind, placebo-controlled dose-finding study. Pain. 2015 Dec;156(12):2438-48. https://doi. org/10.1097/j.pain.0000000000000309 PMID:26230741

46. Trabulsi EJ, Patel J, Viscusi ER, Gomella LG, Lallas CD. Preemptive multimodal pain regimen reduces opioid analgesia for patients undergoing robotic-assisted laparoscopic radical prostatectomy. Urology. 2010 Nov;76(5):1122-4. https://doi.org/10.1016/j. urology.2010.03.052 PMID:20570321

47. Hval K, Thagaard KS, Schlichting E, Raeder J. The prolonged postoperative analgesic effect when dexamethasone is added to a nonsteroidal antiinflammatory drug (rofecoxib) before breast surgery. Anesth Analg. 2007 Aug;105(2):481-6. https://doi. org/10.1213/01.ane.0000267261.61444.69 PMID:17646509

48. Maund E, McDaid C, Rice S, Wright K, Jenkins B, Woolacott N. Paracetamol and selective and non-selective non-steroidal antiinflammatory drugs for the reduction in morphine-related sideeffects after major surgery: a systematic review. Br J Anaesth. 2011 Mar;106(3):292-7. https://doi.org/10.1093/bja/aeq406 PMID:21285082

49. Martínez V, Beloeil $H$, Marret E, Fletcher D, Ravaud P, Trinquart L. Non-opioid analgesics in adults after major surgery: systematic review with network meta-analysis of randomized trials. $\mathrm{Br}$ J Anaesth. 2017 Jan;118(1):22-31. https://doi.org/10.1093/bja/ aew391 PMID:28039239

50. White PF, Sacan O, Tufanogullari B, Eng M, Nuangchamnong $\mathrm{N}$, Ogunnaike B. Effect of short-term postoperative celecoxib administration on patient outcome after outpatient laparoscopic surgery. Can J Anaesth. 2007 May;54(5):342-8. https://doi. org/10.1007/BF03022655 PMID:17470884

51. Aguilera Gómez J.L, Martín Romero, J. L. Delgado Martínez, A. D. Analgesia postoperatoria tras artroplastia total de rodilla. Rev. S. And. Traum. y Ort., 2016; 33 (4/4): 33-46.

52. Kurd MF, Kreitz T, Schroeder G, Vaccaro AR. The role of multimodal analgesia in spine surgery. J Am Acad Orthop Surg. 2017 
Apr;25(4):260-8. https://doi.org/10.5435/JAAOS-D-16-00049 PMID:28291143

53. Rivkin A, Rivkin MA. Perioperative nonopioid agents for pain control in spinal surgery. Am J Health Syst Pharm. 2014 Nov;71(21):1845-57. https://doi.org/10.2146/ajhp130688 PMID:25320134

54. Gilron I, Vandenkerkhof E, Katz J, Kehlet H, Carley M. Evaluating the Association Between Acute and Chronic Pain After Surgery: Impact of Pain Measurement Methods. Clin J Pain. 2017 Jul;33(7):588-94. https://doi.org/10.1097/ AJP.0000000000000443 PMID:28145910

55. Gerbershagen HJ, Ozgür E, Dagtekin O, Straub K, Hahn M, Heidenreich $A$, et al. Preoperative pain as a risk factor for chronic post-surgical pain - six month follow-up after radical prostatectomy. Eur J Pain. 2009 Nov;13(10):1054-61. https://doi. org/10.1016/j.ejpain.2008.11.020 PMID:19138869

56. Schug SA, Bruce J. Estratificación del riesgo para el desarrollo de dolor posquirúrgico crónico. Rep. De dolor 2017; 2 (6): e627. Publicado el 31 de octubre de 2017. https://doi.org/10.1097/ PR9.0000000000000627.

57. Van de Ven T, Hsia J, Lun H. Causes and prevention of chronic postoperative pain, Current opinion in intensive care: August 2012 - Volume 18 - Number 4 - p 366-371 https://doi.
org/10.1097/MCC.0b013e3283557a7f.

58. Huang CC, Sun WZ, Wong CS. Prevention of Chronic Postsurgical Pain: The Effect of Preventive and Multimodal Analgesia. Asian J Anesthesiol. 2018 Sep;56(3):74-82. https://doi. org/10.6859/aja.201809_56(3).0002 PMID:30583329

59. Kopf A, Banzhaf A, Stein CH. (2005). Perioperative management of the patient with chronic pain., 19 (1), 59-76. . Available online at http://www.sciencedirect.com https://doi.org/10.1016/j. bpa.2004.08.002.

60. Vickers AJ, Cronin AM, Maschino AC, Lewith G, MacPherson $\mathrm{H}_{\text {, }}$ Foster NE, et al. Acupuncture for chronic pain: meta-analysis of individual patient data. Arch Intern Med. 2012;172(19):144453. https://doi.org/10.1001/archinternmed.2012.3654 PMID:22965186

61. Dale VH. El impacto de la música perioperatoria en la experiencia del dolor posoperatorio de los pacientes de cirugía abdominal: una revisión sistemática y un metanálisis. Revista de práctica perioperatoria. 2021; 31 (1-2): 31-43. https://doi. org/10.1177/1750458920943375.

62. Bakker CJ, Wise KL, Williams BR, Swiontkowski MF. Complementary and Alternative Medicine for Postoperative Pain: A Systematic Review. J Bone Joint Surg Am. 2020 May;102 Suppl 1:36-46. https://doi.org/10.2106/JBJS.19.01439 PMID:32251133 